Purpose To describe and evaluate this process and the efficiency of quality indicators.

Materials and Methods Prospective study. Period: 1 year (2011). Academic General Hospital (1,500 beds). Antifungal drugs: liposomal amphotericin B, anidulafungin, caspofungin, voriconazole. Variables: process and efficiency quality indicators. Data source: daily log sheet preparation and return of IVM, and antifungal and infusion solution direct costs; personnel costs weren't considered. Results 3,643 antifungal IVMs were prepared: $35 \%$ caspofungin, $32 \%$ voriconazole, $21 \%$ liposomal amphotericin B, $12 \%$ anidulafungin. Process QI: $6.40 \%$ antifungal IVMs returned (mainly voriconazole: $10 \%$ ) and $87 \%$ antifungal IVMs recycled (mainly caspofungin: $100 \%)$. Total savings: $€ 222,351$. Efficiency of the OIs: $€ 155,694$ savings from $\mathrm{PhDp}$ centralization (mainly voriconazole: $€ 78,659$ ) and $€ 66,657$ savings from recycling (mainly caspofungin: $€ 33,025$ ).

Conclusions The fact that process quality indicators comply with standards and the very large cost savings for the institution, support $\mathrm{PhDp}$ antifungal IVM centralization. Voriconazole IVM centralization allows more cost savings and caspofungin is the most recycled.

No conflict of interest.

\section{OHP-032 EMTRICITABINE AND TENOFOVIR DISOPROXIL FUMARATE IN HIV-NAIVE PATIENTS: A PHARMACOECONOMIC STUDY}

doi:10.1136/ejhpharm-2013-000276.406

${ }^{1} \mathrm{G}$ Nota, ${ }^{1} \mathrm{~S}$ Cirillo, ${ }^{2} \mathrm{G}$ Cinnirella, ${ }^{3} \mathrm{~A}$ Leggieri, ${ }^{4} \mathrm{~L}$ Poggio, ${ }^{5} \mathrm{~L}$ Cattel. ${ }^{1}$ Università degli Studi di Torino, Scuola di Specializzazione in Farmacia Ospedaliera, Turin, Italy; ${ }^{2}$ Presidio Ospedaliero Amedeo di Savoia Birago di Vische ASLTO2, Farmacia interna, Turin, Italy; ${ }^{3}$ Ospedale M.Vittoria - Ospedale S.G.Bosco ASLTO2, S.C. Farmacia Ospedaliera, Turin, Italy; ${ }^{4}$ ASL TO5, S.C. Farmacia Ospedaliera, Turin, Italy; ${ }^{5}$ Università degli Studi di Torino, Dipartimento di Scienza e Tecnologia del Farmaco, Turin, Italy

Background Truvada, a fixed-dose combination of antiretroviral drugs (emtricitabine and tenofovir fumarate) indicated for HIV-1, was the 12th most expensive drug prescribed in Piedmont during 2009-2010, with a growth of $12 \%$.

Since July 2011 the School of Hospital Pharmacy in Turin has developed a two-year pharmacoeconomic project regarding highcost drugs.

Purpose To provide to the decision-makers with a management tool to evaluate the treatment costs of HIV patients.

Materials and Methods The legislation and articles in epidemiology and pharmacoeconomic journals were reviewed. Drummond's Weighted Checklist method was used to evaluate the pharmacoeconomic articles. A Budget Impact model, based only on the drug costs, was built. The treatment-naive population (290) was extrapolated from the incidence data in Piedmont in 2010. The treatment options relied on the US Department of Health and Human Services guidelines and on the pharmacoeconomic studies. The model suggested a combination of Truvada with: i) efavirenz (NNRTI, Sustiva), ii) atazanavir (PI, Reyataz) + ritonavir (PI booster, Norvir); iii) darunavir (PI, Prezista) + ritonavir (PI booster, Norvir).

Results The daily treatment cost for a treatment-naive patient varies from $€ 21.78$ to $€ 30.64$, while the annual expenditure varies from $€ 7,949.17$ to $€ 11,184.45$. The Budget Impact was calculated assuming that the 290 new HIV cases had been treated for one year with one of the therapeutic strategies provided. The variation in comparison with association i) were respectively $+24.64 \%$ for combination ii) and $+40.70 \%$ for combination iii). Treatment iii) was the most expensive $(€ 324,3491.37)$ and increased the annual expenditure by $40.70 \%(€ 938,233.23)$ as compared with treatment i) $(€ 2305,258.14)$.

Conclusions The Budget Impact analysis will be used to perform pre-assessments of expenditure in order to set up health care programmes for the allocation of the economic resources.
A pharmacoeconomic analysis of cost-effectiveness will be performed between the associations Truvada + Reyataz and Truvada + Sustiva.

No conflict of interest.

\section{OHP-033 EPIDEMIOLOGICAL STUDY OF INTOXICATIONS BY ALCOHOL AND DRUG ABUSE IN THE EMERGENCY DEPARTMENT OF LUGO HOSPITAL IN 2009}

doi:10.1136/ejhpharm-2013-000276.407

${ }^{1} \mathrm{AM}$ Bermejo Barrera, ${ }^{2} \mathrm{Al}$ Cachafeiro Pin, ${ }^{2} \mathrm{AM}$ López-Vizcaino castro, ${ }^{2 P}$ Sempere Serrano, ${ }^{3} \mathrm{MD}$ Formoso Lavandeira, ${ }^{2} \mathrm{~V}$ López García. ${ }^{1}$ Hospital Lucus Augusti, Toxicología USC, Santiago de Compostela, Spain; ${ }^{2}$ Hospital Lucus Augusti, Servicio de Farmacia, Lugo, Spain; ${ }^{3}$ Hospital Lucus Augusti, Servicio de Análisis Clinicos, Lugo, Spain

Background Acute poisoning is a condition that generates great demand for care in emergency departments of hospitals.

Purpose To find out the epidemiology of severe acute intoxications and study the profile of the intoxicated patients in our hospital.

Materials and Methods Retrospective observational study. Inclusion criteria: patients with final diagnosis of acute intoxication during 2009. Sources: admission management software, clinical histories. Data recorded: age, sex, date of entry, type of toxic agent(s) involved, existence of psychiatric background and previous intoxications. Global analysis: SPSS package.

Results During the study period 1052 requests for analysis were processed with the following results: (see the table below)

\section{Abstract OHP-033 Table 1}

\begin{tabular}{|c|c|c|c|c|}
\hline $\begin{array}{l}\text { Drugs } \\
\text { (\% positive) }\end{array}$ & $\begin{array}{l}\text { Sex } \\
\text { distribution } \\
\text { (\% men) }\end{array}$ & $\begin{array}{l}\text { Band } \\
\text { age } \\
\text { (years) }\end{array}$ & $\begin{array}{l}\text { Majority of } \\
\text { intoxications by day } \\
\text { of the week (DW) }\end{array}$ & $\begin{array}{l}\text { Months of the year } \\
\text { with highest numbers } \\
\text { of positives (M) }\end{array}$ \\
\hline Ethyl alcohol (65\%) & $80 \%$ & $50-59$ & Sunday & August, June, November \\
\hline Benzodiazepines (36\%) & $50 \%$ & $40-49$ & Thursday & June and August \\
\hline Cannabis (12\%) & $80 \%$ & $20-29$ & $\begin{array}{l}\text { Sunday }>\text { Friday }> \\
\text { Saturday }\end{array}$ & June and August \\
\hline Cocaine (6\%) & $80 \%$ & $30-39$ & weekend consumption & August \\
\hline Opiates (5.4\%) & $81 \%$ & $30-39$ & weekend consumption & April $=$ August \\
\hline
\end{tabular}

Amphetamines (0.19\%): 2 men under the age of 20 and 30 years, M: January, DW: weekend. Barbiturates (0.38\%): 4 positive, $75 \%$ men, A: 42-54. A temporal distribution (week, month year day) cannot be significant in so few cases.

Conclusions For a better understanding of the Spanish reality in terms of acute intoxication referrals, systematic multi-centre, clinical and epidemiological studies are necessary to demonstrate changes in the toxic substance used, the distribution by age, characteristics of subjects, etc. In order to adapt the health care resources, we need to know the diagnosis and any treatment that would contribute to improving the care of intoxicated patients. See table.

No conflict of interest.

\section{OHP-034 ESTIMATION OF THE ADHERENCE TO BIOLOGICAL TREATMENT IN PATIENTS WITH PSORIASIS}

doi:10.1136/ejhpharm-2013-000276.408

I Loizaga-Diaz, Z Perez-Espana, S Vallinas-Hidalgo, A Llona-Armada, FJ GoikoleaUgarte, AV Aguirrezabal-Arredondo, MJ Yurrebaso-lbarreche. Basurto University Hospital, Pharmacy Service, Bilbao, Spain

Background Biological agents have changed the treatment of psoriasis, and are used for long-term treatment. For this reason adherence to the treatment is a marker of success.

Purpose To quantify the adherence of patients with psoriasis to treatment with biologicals (adalimumab, etanercept and infliximab) Materials and Methods Retrospective observational study of psoriasis patients who were prescribed biologicals. Sex, age, type of 
biological agent, dose and adherence were examined. To calculate the adherence we used a record of prescriptions dispensed over a period of six months. We used the formula: \% adherence $=$ no. of units dispensed/no. of units theoretically needed $\times 100$.

Results The sample included 62 patients, 45 males and 17 females with mean age of 50 years (range 12-81). 53.2\% were using etanercept, $43.6 \%$ adalimumab and $3.2 \%$ infliximab. The adherence was high in the infliximab group (94\%) and very similar in the other groups (etanercept $83.7 \%$, adalimumab $87.4 \%$ ). In the adalimumab cohort $11 \%$ had a reduced dose, in the etanercept group 9\% had a reduced and $30 \%$ an increased dose. In all these groups the calculated adherence was quite similar.

Conclusions As described in the literature, adherence to biologicals was significantly higher compared with the adherence observed with other treatments for psoriasis. Infliximab had the highest rate, maybe because it is administered in hospital. There was no difference between adalimumab and etanercept. It is known that there is progressive loss of patient adherence to treatment, for this reason is important to focus the attention on this concept.

No conflict of interest.

\section{OHP-035 EVALUATING SINGLE-INCISION SLINGS IN FEMALE STRESS URINARY INCONTINENCE: THE USEFULNESS OF THE CONSORT STATEMENT CRITERIA}

doi:10.1136/ejhpharm-2013-000276.409

$\underline{{ }^{1} \mathrm{~S} \text { Diallo, }}{ }^{1} \mathrm{~A}$ Josephson, ${ }^{2} \mathrm{~F}$ Cour, ${ }^{2} \mathrm{~A}$ Vidart, ${ }^{2 \mathrm{H}}$ Botto, ${ }^{2 \mathrm{~T}}$ Lebret, ${ }^{1 B}$ Bonan. ${ }^{1} \mathrm{Hôpital}$ Foch, Pharmacy, Suresnes, France; ${ }^{2}$ Hôpital Foch, Urology, Suresnes, France

Background Unlike drugs, medical devices (MDs) are not submitted for health authority marketing authorization based on in-depth clinical evaluation: critical review on an evidence-based medicine approach is essential for practitioners. The Consolidated Standards of Reporting Trials (CONSORT) statement is an international consensus expert guideline aimed at improving the reporting quality of clinical trials reports.

Purpose To evaluate the usefulness and applicability of the CONSORT for journal articles reporting randomised controlled trials (RCTs) evaluating an implantable $\mathrm{MD}$.

Materials and Methods Original articles published before 2012 reporting RCTs assessing single-incision slings (SISs) in the treatment of female stress urinary incontinence were searched for in PubMed and Embase databases. Reporting quality was assessed by two hospital pharmacists and two urological surgeons according to three CONSORT checklists: abstract (17 items), standard (37 items) and extension for non-pharmacological trials (20 items); the results were discussed to reach a consensus.

Results Among 135 articles retrieved, eight articles met the inclusion criteria and were assessed. Abstract scores ranged from 4.7 to 14.1 out of 20 . Standard scores were greater than 10.0 out of 20 for most articles; the extension scores did not exceed 5.0 out of 10 . Half the reported trials were not identified as randomised in the title. Three articles did not mention any confidence interval or standard deviation for outcomes. The interventions were incompletely described; only four articles reported the configuration of the devices. Four articles reported whether blinding was achieved but lack of blinding was never discussed as a potential source of bias. Few articles reported the operators and centres' characteristics and their impact on statistical analysis.

Conclusions The reporting quality of SISs RCTs should be improved because readers require complete, clear and transparent information to assess the relevance and applicability of results. Our study supports further use of the CONSORT criteria to enhance and assess the reporting quality of surgical trials.

No conflict of interest.

\section{OHP-036 EVALUATION OF ANTIBIOTIC APPRIOPRIATENESS AND USE IN IMOLA HOSPITAL}

doi:10.1136/ejhpharm-2013-000276.410

MC Silvani, L Paternoster, S Calabria, I Martens, S Gambetti. AUSL Imola, Assistenza Farmaceutica, Imola (Bologna), Italy

Background Misuse of antibiotics in hospitals may cause bacterial resistance as well as increased costs and unnecessary exposure of patients to drugs.

Purpose To evaluate antimicrobial consumption and appropriateness through a new antibacterial stewardship policy.

Materials and Methods The study was carried out in Imola Hospital (Bologna) and 2009-2011 drug consumption data were obtained from the pharmacy service. Data were analysed by clinical area and single wards and were expressed by ATC classification and defined daily doses per 100 bed-days (DDD). A form for personalised antibacterial treatment (ATf), including diagnosis and documented reasons for the choice of antibiotic, was introduced for levofloxacin, teicoplanin, meropenem, linezolid, tigecycline and daptomycin.

Results In 2011, overall antibacterial consumption was 78 DDD (+4\% vs. 2010); the major increase was observed in medical units (MED: $+9 \%$ ) and paediatric/gynaecological units $(+6 \%)$. Intensive care units/emergency department (ICUs/EDs) and surgical units (SUR) exhibited a decrease in consumption $(-13 \%,-7 \%$, respectively). The use of critical antimicrobial agents decreased: fluoroquinolones (19 DDD, -15\%), carbapenems (3.5 DDD, -18\%) and glycopeptides (3.1 DDD, $-17 \%$ ). The introduction of ATfs (May 2011) contributed to a decrease in the consumption of antibiotics (e.g. MED: 75 DDD semester I vs. 71 DDD semester II 2011; overall 2011: 73 DDD). The analysis of ATfs shows that critical antibacterial agents were mainly prescribed to treat respiratory tract infections (MED: 58\%, ICU/ED: 44\%, SUR 30\%), urinary tract (MED e ICU/ ED: 20\%), skin and soft tissues (SUR: 35\%, ICU/ED: 16\%, MED: 6\%) and intra-abdominal infections (SUR: $9 \%$ ). Levofloxacin (55\%) and meropenem $(11 \%)$ were the most prescribed for respiratory tract infections, teicoplanin (6\%) for skin and soft tissue infections.

Conclusions Our stewardship policy led to a reduction in the use of wide-spectrum antibiotics, so ATf may represent a valid method of rationalising the choice of antimicrobial treatment.

Acknowledgements Agenzia Italiano Del Farmaco, Emilia Romagna Region.

No conflict of interest.

\section{OHP-037 EVALUATION OF CHANGE OF ETANERCEPT SUBCUTANEOUS ADMINISTRATION DEVICE}

doi:10.1136/ejhpharm-2013-000276.411

${ }^{1} \mathrm{MJ}$ Gayan Lera, ${ }^{1} \mathrm{M}$ Ercilla Liceaga, ${ }^{1} \mathrm{C}$ Ripa Ciaurriz, ${ }^{2} \mathrm{C}$ Sarasqueta Eizaguirre, ${ }^{1} \mathrm{I}$ Barral Juez, 'MD Mauleon Echeverria, "I Aguirre Zubia, 'P Pascual Gonzalez, 'P Carmona Oyaga, 'M Umerez Igartua. 'Donostia University Hospital, Pharmacy, San Sebastián, Spain; ${ }^{2 B i o d o n o s t i a ~}$

Background Etanercept is a soluble tumour necrosis factor receptor fusion protein used in a variety of arthropathies. A new administration device (pen) has recently been marketed.

Purpose To evaluate pain differences and preference between the etanercept syringe and pen as well as the relation between pain and demographic and anthropometric factors.

Materials and Methods All patients with the etanercept pen from 1 January 2012 to 31 March 2012 who had previously used the syringe were chosen. Gender, age, Body Mass Index (BMI), diagnosis, self-administration, pain perception $(0=$ no pain; $10=$ maximum pain) and device preference were recorded. Statistical analysis: Student's t-test and variance analysis were used for comparisons of means, chi-square and Fisher's test for proportions, and nonparametric tests for pain. 